\title{
Ambiente de aprendizaje positivo, compromiso académico y aprendizaje autorregulado en bachilleres
}

\author{
José Concepción Gaxiola Romero; Eunice Gaxiola Villa; Nadia Saraí Corral Frías; Paola Escobedo Hernández
}

How to cite this article:

Gaxiola Romero, J.C., Gaxiola Villa, E., Corral Frías, N.S., \& Escobedo Hernández, P. (2020). Positive learning environment, academic engagement and self-regulated learning in high school students. Acta Colombiana de Psicología, 23(2), 267-278. http://www.doi.org/10.14718/ACP.2020.23.2.11

Recibido, agosto 10/2019; Concepto de evaluación, diciembre 12/2019; Aceptado 10 marzo 2020

\author{
José Concepción Gaxiola Romero* \\ Universidad de Sonora, Sonora, México \\ ORCID: https://orcid.org/0000-0001-8037-3082 \\ Eunice Gaxiola Villa \\ Universidad de Sonora, Sonora, México \\ ORCID: https://orcid.org/0000-0003-4673-7094 \\ Nadia Saraí Corral Frías \\ Universidad de Sonora, Sonora, México \\ ORCID: https://orcid.org/0000-0002-1934-0043 \\ Paola Escobedo Hernández \\ Universidad de Sonora, Sonora, México \\ ORCID: https://orcid.org/0000-0001-9175-6521
}

\begin{abstract}
Resumen
De acuerdo con la teoría de ambientes positivos, los contextos donde se brinda apoyo académico pueden promover la adaptación exitosa de los estudiantes, y, a su vez, el resultado de dicha adaptación favorece su éxito académico, lo cual puede medirse con los constructos de compromiso académico y aprendizaje autorregulado en el ámbito académico. Teniendo esto en consideración, en la presente investigación se da cuenta de un trabajo de psicología positiva aplicada en el que se tuvo como objetivo evaluar la relación entre el ambiente de aprendizaje positivo (AAP), el compromiso académico y el aprendizaje autorregulado en bachilleres mexicanos. Para esto, se diseñó un estudio de tipo correlacional en el que participaron 166 estudiantes voluntarios de primer grado de bachillerato, seleccionados por conglomerados, con consentimiento informado individual y de sus padres. Del total de participantes, 76 fueron de sexo masculino (45.8 \%) y 90 de sexo femenino $(54.2 \%)$, con una edad promedio de 15.2 años $(D E=.43)$; quienes respondieron a cuestionarios sobre apoyo académico de padres, amigos pro-académicos, ambiente familiar positivo, compromiso académico y aprendizaje autorregulado. Se realizó un análisis de ecuaciones estructurales, y en los resultados se encontró, con una $p<.05$, que el AAP fue un factor latente formado por el apoyo académico de los padres, los amigos pro-académicos y el ambiente familiar positivo; además, en el modelo estructural, el AAP correlacionó positivamente con el compromiso académico (coeficiente estructural $=.80$ ), y este último se relacionó positivamente con el aprendizaje autorregulado de los estudiantes (coeficiente estructural $=.55$ ); finalmente el modelo estructural resultante explicó el $30 \%$ del aprendizaje autorregulado. Para concluir, se discute respecto a la posibilidad de que el AAP promueva el compromiso académico y el aprendizaje autorregulado de los estudiantes.

Palabras clave: ambientes positivos, éxito académico, adolescentes, aprendizaje autorregulado.
\end{abstract}

\footnotetext{
* Blvd. Luis Encinas y Rosales S/N, Colonia Centro (C.P.: 83000), Hermosillo, Sonora, México. Tel.: (52) 662 2592173. jose.gaxiola@unison.mx

El presente artículo se realizó gracias al financiamiento del proyecto CONACYT, clave 281010, "Variables ecológicas predictoras de la resiliencia académica: un estudio longitudinal".
} 


\title{
Positive learning environment, academic engagement and self-regulated learning in bigh school students
}

\begin{abstract}
From the perspective of positive environment theory, supportive environments, can promote student successful academic adaptation. Accordingly, student academic success is the result of academic adaptation, and can be assessed via means of academic engagement and self-regulated learning. Thus, the aim of the present study was to test the relationship between positive learning environments (POLE), academic engagement and self-regulated learning in high school students. Using a cross-sectional design, participants included 166 freshmen high school students, 76 participants of the sample were male $(45.8 \%)$ and 90 were female $(54.2 \%)$. Mean age was $15.2($ S.D. $=.43)$. Before participation informed consent was obtained from parents and assent from participants. Students answered a questionnaire regarding academic support given by parents, pro-academic friends, positive family environment, and academic engagement and self-regulated learning. Using Structural Equation Modeling the results demonstrated $(p<.05)$ that POLE represented a latent factor formed by parental academic support, pro-academic friends and positive family environment. The structural model showed that POLE was positively related to academic engagement (structural coefficient $=.80$ ); also, academic engagement was related with self-regulated learning (structural coefficient $=.55$ ) and explained $30 \%$ of student self-regulated learning. Results suggest that POLE could promote academic engagement and self-regulated learning.

Keywords: positive environments, academic success, adolescents, self-regulated learning.
\end{abstract}

\section{Introducción}

En México, el abandono escolar, en especial el que se presenta en el nivel medio superior -0 bachillerato-, constituye un serio problema para el sistema educativo básico nacional. De hecho, en el año 2017, la eficiencia terminal en el nivel de bachillerato fue del $67.3 \%$ (INEE, 2017), lo que implica que el $32.7 \%$ de los estudiantes que ingresaron a este nivel no concluyeron sus estudios.

A partir de la literatura científica sobre el tema se ha podido identificar que, por una parte, el abandono escolar en el nivel medio superior puede ser consecuencia de situaciones complejas de tipo multifactorial — donde participan factores psicológicos, sociales, económicos y culturales-; $\mathrm{y}$, por otra, que desde la perspectiva psicológica existen dos variables relacionadas con el desempeño y la permanencia académica exitosa de los estudiantes de bachillerato, a saber: el compromiso académico (Gómez et al., 2015) y el aprendizaje autorregulado de los estudiantes (Chase et al., 2014; Daura, 2015); que también son considerados como factores protectores del abandono escolar de los estudiantes.

En la presente investigación se toma como base el marco conceptual que proporciona la psicología ambiental positiva (Corral et al., 2014); teoría en la que se propone la existencia de instituciones positivas (Seligman \& Csikszentmihalyi, 2000) en las cuales un ambiente de aprendizaje positivo constituye entornos sustentables de apoyo académico que promueven el ajuste escolar de los estudiantes -identificable a partir de los constructos de compromiso académico y autorregulación del aprendizaje-. En este sentido, en los ambientes de aprendizaje positivo la inversión en apoyo académico que realizan las personas cercanas a los estudiantes - como padres, maestros y amistades - obtiene como resultado el ajuste escolar y el bienestar psicológico de todos los implicados, lo cual representaría la base de la sustentabilidad del sistema, es decir, garantizar la permanencia de los apoyos y de los resultados académicos en el tiempo.

En la psicología ambiental positiva, los ambientes positivos - o entornos sustentables - suelen propiciar la adaptación psicológica de las personas que interactúan en ellos. De hecho, se ha encontrado que en dichos contextos las familias positivas tienen un papel fundamental, pues en ellas se administra equitativamente lo concerniente a los recursos físicos y psicológicos para cada uno de sus miembros, además de que se propicia el desarrollo y el crecimiento óptimo de las personas que viven y se desenvuelven en ellas (Corral et al., 2014).

En este sentido, las familias positivas manifiestan una serie de características transaccionales en las que sus miembros dan y reciben recursos para facilitar el mantenimiento de la familia, dentro de las cuales se pueden encontrar, por ejemplo, (a) las transacciones afectivas, que incluyen dar y recibir afecto a los miembros de la familia; (b) las transacciones educativas, entendidas como intercambios de conocimiento y habilidades entre la familia; 
(c) las transacciones cooperativas, que consisten en dar y recibir apoyos diversos para completar tareas; y (d) las transacciones económicas, que refieren a los intercambios entre los miembros para el mantenimiento y desarrollo financiero de toda la familia (Corral et al., 2014).

Ahora bien, en la literatura relacionada se encuentra también una correlación importante entre las familias que proporcionan un entorno de comunicación y cooperación entre sus miembros y el compromiso académico de los estudiantes (Eccles \& Harold, 2013); así como que los padres que apoyan las actividades escolares de sus hijos -brindándoles recursos materiales y motivacionales para su educación - pueden mejorar el compromiso académico de los más jóvenes (Álvarez et al., 2015). De hecho, el compromiso académico también se ha relacionado con tener amistades pro-académicas, es decir, amistades que otorgan recursos emocionales e instrumentales para el fortalecimiento del aprendizaje de sus amigos (Chen, 2008; Shin \& Ryan, 2014), puesto que estas pueden afectar su motivación y persistencia académica (Ricard \& Pelletier, 2016; Rodríguez et al., 2018).

En este sentido, de acuerdo con la revisión anterior, y con base en la propuesta teórica de ambientes positivos (Corral et al., 2014), el ambiente de aprendizaje positivo puede caracterizarse por amistades pro-académicas, un ambiente familiar positivo y el apoyo académico de los padres; variables que, como se trata a continuación, correlacionan directamente con el compromiso académico e indirectamente con el aprendizaje autorregulado.

En particular, el compromiso académico incluye aspectos institucionales - como reglas, instalaciones y horarios de la escuela-, así como el esfuerzo del estudiante por aprender, y sus sentimientos de seguridad o conformidad dentro de su escuela (Yazzie-Mintz, 2009). Incluso, dentro de las dimensiones del compromiso académico se pueden encontrar: (a) el compromiso emocional, entendido como el interés, la actitud positiva hacia el aprendizaje y el sentido de pertenencia; (b) el compromiso conductual, que refiere al esfuerzo, la participación y la conducta positiva del estudiante; y (c) el compromiso cognitivo, es decir, metas de aprendizaje e inversión cognitiva en el aprendizaje (Appleton et al., 2006; Fredericks et al., 2004).

Por su parte, el aprendizaje autorregulado se ha identificado como un aspecto de gran importancia en la educación de los estudiantes, debido a que, por una parte, promueve en ellos la conciencia de su pensamiento, y, por otra, incentiva el hecho de tener estrategias, aprender a direccionar su motivación y dirigir sus esfuerzos a metas importantes que mejoren su rendimiento académico (Lamas, 2008). De acuerdo con esto, los estudiantes que emplean el aprendizaje autorregulado usan de manera efectiva sus estrategias de aprendizaje, evalúan sus progresos y son persistentes en la ejecución de comportamientos dirigidos hacia su meta (Zimmerman, 2000, 2002). Adicional a esto, investigaciones recientes señalan que los alumnos que utilizan estrategias de aprendizaje autorregulado, donde establecen metas académicas y estrategias para alcanzarlas, obtienen mejores resultados en sus materias (Roux, 2015); además de que hay un efecto positivo de las estrategias de aprendizaje sobre el rendimiento académico (Daura, 2015).

Ahora, de acuerdo con Sharma (2013), el aprendizaje autorregulado incluye: (a) habilidades de organización, que posibilitan la selección de las ideas principales y su diferenciación de la información irrelevante; (b) pensamiento crítico, que consiste en la utilización de habilidades y conocimientos previos para pensar lógicamente acerca de nuevas situaciones; (c) estrategias de elaboración, que refieren al uso de estrategias de aprendizaje, como parafrasear, resumir y tomar notas, que implican la traducción - con palabras propias - del conocimiento nuevo al ya existente; y (d) autorregulación metacognitiva, que incluye la planeación de metas o actividades, el monitoreo de las acciones que promueven la atención, la autoevaluación del progreso, y la regulación continua de las estrategias cognitivas y metas.

Cabe mencionar que los estudios sobre el aprendizaje autorregulado parten de la teoría sociocognitiva del aprendizaje, donde las características de los apoyos otorgados promueven y mantienen los comportamientos académicos de los estudiantes (Zimmerman, 2002); y que investigaciones longitudinales aportan evidencia sobre la importancia de los ambientes de apoyo que rodean a los estudiantes - como los padres y amigos - en el desarrollo del aprendizaje autorregulado (King \& Ganotice, 2012; Wang \& Eccles, 2012). Incluso, diversos estudios indican la posibilidad de que el compromiso académico preceda al aprendizaje autorregulado, al ser un elemento motivador que incentiva el desarrollo y el mantenimiento personal de las ejecuciones académicas a largo plazo (Skinner \& Pitzer, 2012); y, relacionado con esto, se podría afirmar que el aprendizaje autorregulado requiere como antecedentes que los estudiantes perciban control y aceptación de su entrono académico y de sus emociones relacionadas con el ámbito escolar, lo cual sería un indicador del compromiso académico emocional y cognitivo (You \& Kang, 2014).

Adicional a esto, investigaciones sobre los ambientes de aprendizaje positivo desarrolladas en México y con distintas muestras de estudiantes de bachillerato señalan una relación directa entre, por una parte, la percepción de apoyo de los padres, las amistades y el ambiente familiar positivo, y, por otra, la autorregulación académica de los 
estudiantes. De hecho, en algunos estudios se reporta una relación indirecta entre estas variables mediada por las disposiciones a la resiliencia y las metas académicas (Gaxiola \& González, 2019), así como una relación indirecta entre el aprendizaje positivo y el compromiso académico (Gaxiola et al., 2018).

Por otra parte, conocer las variables ambientales relacionadas con el aprendizaje autorregulado y el compromiso académico de los estudiantes de bachillerato en el contexto latinoamericano e internacional puede servir de base para el desarrollo de futuros programas de promoción, prevención y rehabilitación dirigidos a mejorar el ajuste académico y la permanencia exitosa de los estudiantes en sus escuelas. Anuado a lo anterior, a nivel disciplinario, en psicología resulta de gran importancia justificar empíricamente la relevancia de los ambientes de aprendizaje positivos en las variables relacionadas con el ajuste académico de los estudiantes de bachillerato.

Teniendo esto en cuenta, el objetivo de la presente investigación fue evaluar la relación existente entre el ambiente de aprendizaje positivo, el compromiso académico y el aprendizaje autorregulado de estudiantes de bachillerato; siendo el ambiente de aprendizaje positivo la variable independiente exógena, el compromiso académico la variable independiente mediadora, y el aprendizaje autorregulado la variable dependiente a evaluar. Como hipótesis, se planteó que el ambiente de aprendizaje positivo se relacionaría positivamente con el compromiso académico, y que este, a su vez, presentaría una relación positiva con el aprendizaje autorregulado. El modelo hipotético se presenta en la Figura 1.

\section{Método}

\section{Diseño}

Se realizó una investigación de tipo correlacionaltransversal, y en la selección de la muestra se utilizó un diseño por conglomerados.

\section{Participantes}

Los participantes fueron estudiantes del bachillerato que obtuvieron niveles de aprovechamiento cercanos al promedio en la prueba oficial PLANEA, aplicada en la ciudad de Hermosillo, Sonora, en México (SEP, 2017), una encuesta nacional que mide anualmente el nivel de aprovechamiento estudiantil en materias como matemáticas, lenguaje y comunicación.

Para la selección de la muestra se utilizó como criterio contactar a una escuela de bachillerato con nivel promedio de aprovechamiento, para así evitar posibles sesgos de aprendizaje autorregulado o de compromiso académico en estudiantes pertenecientes a instituciones educativas con muy bajos o muy altos logros de aprovechamiento escolar. De este modo, en la escuela seleccionada solo el $20 \%$ de

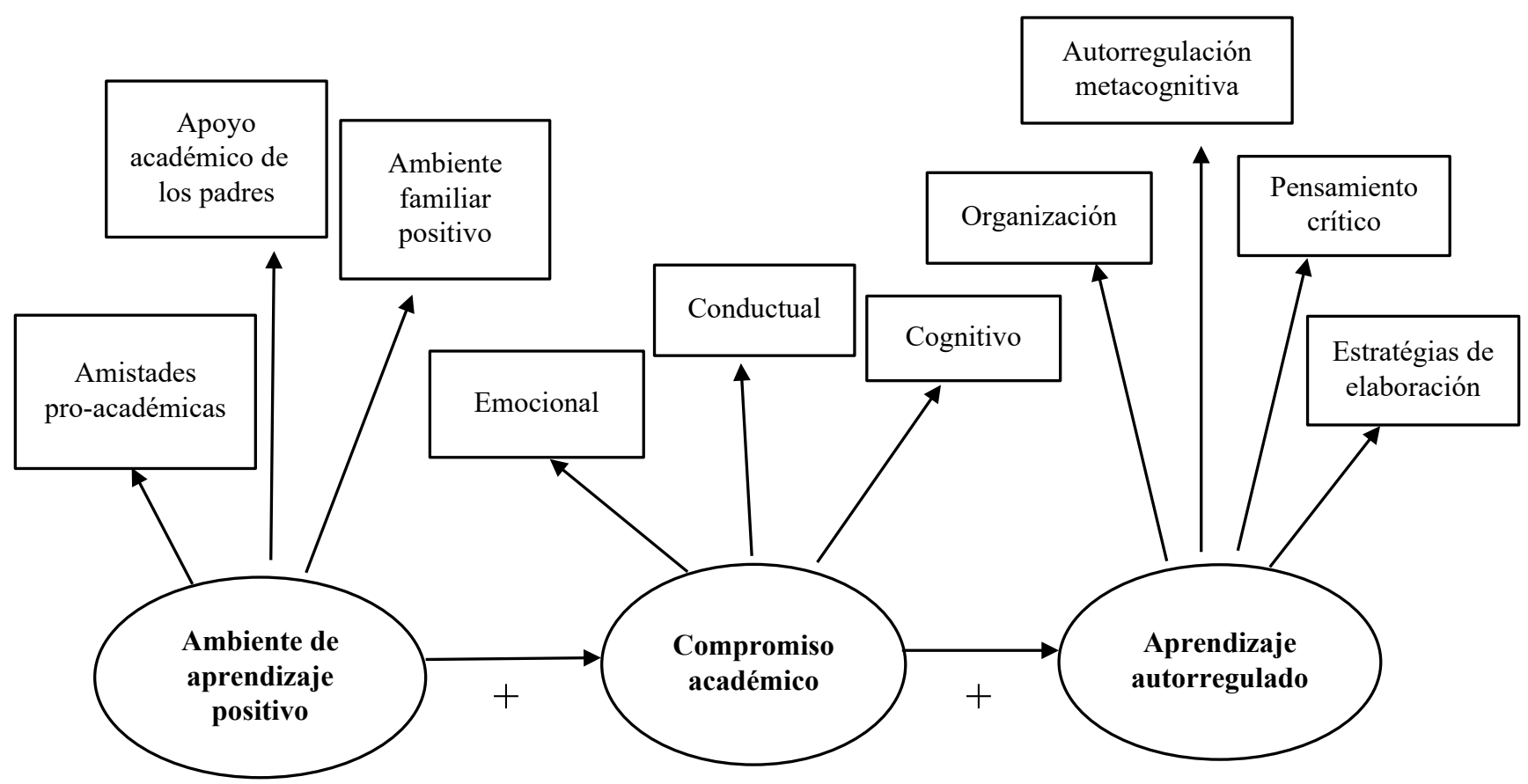

Figura 1. Modelo hipotético de aprendizaje autorregulado con la mediación del compromiso académico. 
los estudiantes puntuaron en el nivel más avanzado de logro (nivel IV) en el área de lenguaje y comunicación; mientras que solo el $14.3 \%$ se ubicó en el nivel más avanzado (nivel IV) en el área de matemáticas.

Después de seleccionar la escuela, con el cuidado de los principios éticos de confidencialidad y con la firma del consentimiento informado de manera individual y por parte de los padres, se invitó a participar voluntariamente a todos los estudiantes del primer año de bachillerato en la investigación; esto con el fin de incluir en la muestra a la mayor cantidad de estudiantes de nuevo ingreso antes de que alguno decidiera abandonar sus estudios. Posteriormente, para calcular del tamaño de la muestra necesario para la investigación se utilizó el software para el cálculo del tamaño de muestra a-priori para modelos de ecuaciones estructurales de Soper (2018); análisis que se desarrolló con la consideración de un modelo hipotético compuesto por tres variables latentes, diez variables manifiestas, un tamaño de efecto anticipado entre los parámetros alto (.5), un nivel de poder estadístico de 9 , y un nivel de probabilidad esperado de .05. De este cálculo resultó la necesidad de seleccionar una muestra de al menos 156 participantes.

Finalmente, para la selección de los conglomerados, y con el fin de cumplir con el tamaño de muestra recomendado, se seleccionaron, con el uso de números aleatorios, cinco grupos al azar de un total de ocho grupos de primer ingreso, formados con un promedio de 40 alumnos por grupo. De los conglomerados elegidos participaron 166 estudiantes en total, de los cuales el $45.8 \%(\mathrm{n}=76)$ era de sexo masculino, y el $54.2 \%(\mathrm{n}=90)$ de sexo femenino. La edad promedio de los estudiantes fue de 15.2 años $(D E=0.43)$.

\section{Instrumentos}

Los instrumentos aplicados se compilaron en un cuadernillo que incluyó preguntas sobre datos sociodemográficos como edad y sexo, además de escalas tipo Likert para medir cada una de las variables de la investigación.

En particular, con el fin de evaluar las amistades proacadémicas se aplicó la traducción del friend-peer academic support scale (FPASS) de Chen (2005), una escala de 22 preguntas que mide los apoyos emocionales e instrumentales relacionados con las actividades académicas realizadas por los amigos (p. ej., "Mis amigos están dispuestos a ayudarme a aprender"), cuyas opciones de respuesta se presentan en una escala tipo Likert de seis puntos - de 0 (nunca) a 5 (siempre) —. Para este instrumento, su autor (Chen, 2008) reportó una consistencia interna alta $(\alpha=.83)$.

Por otra parte, el apoyo académico de los padres se midió con la traducción de la parental academic support scale (PASS), de Chen (2005); una escala de 28 preguntas sobre el apoyo académico instrumental y emocional de los padres (p. ej., "Mis padres me compran materiales de aprendizaje complementarios"). De igual forma, las preguntas se responden en una escala Likert de seis puntos - de 0 (nunca) a 5 (siempre)-, y el instrumento obtuvo una consistencia interna alta $(\alpha=.88)$ (Chen, 2008).

Adicional a esto, para evaluar el ambiente familiar positivo se utilizó la escala de familias positivas (FAMPOS), de Aranda et al. (2015), un instrumento de 19 reactivos que evalúa las transacciones afectivas, educativas, cooperativas y económicas que mantienen los integrantes de la familia (p. ej., "Expresamos cariño entre nosotros"). Estas preguntas se responden con una escala tipo Likert de cinco puntos de 0 ("nunca") a 4 ("siempre")—; y el instrumento, de acuerdo con los autores (Aranda et al., 2015), presenta una consistencia interna alta $(\alpha=.93)$.

El compromiso académico se midió con la traducción al español de la high school survey of student engagement (HSSSE), de la Universidad de Indiana (Yazzie-Mintz, 2009), un instrumento que evalúa con un total de 121 preguntas el compromiso emocional - en una escala Likert de 1 (fuertemente de acuerdo) a 4 (fuertemente en desacuerdo)-, así como el compromiso conductual —en una escala de 0 (nunca) a 4 (frecuentemente) - y el compromiso cognitivo en una escala de 0 (nunca) a 4 (frecuentemente) y de 1 (totalmente de acuerdo) a 4 (totalmente en desacuerdo)Aunque los autores del inventario no reportaron los valores de confiabilidad del instrumento, Fooladvand et al. (2012) obtuvieron un valor alfa de .96 para el inventario en su conjunto. Acá, un ejemplo de un ítem para el compromiso académico emocional es "Me tratan bien en la escuela", mientras que el compromiso académico conductual incluyó preguntas como "¿Qué tan frecuente hablas con el maestro acerca del trabajo de clase?", y en el compromiso académico cognitivo se incluyeron afirmaciones como "Pongo una gran cantidad de esfuerzo al hacer mi trabajo escolar".

Finalmente, para evaluar el aprendizaje autorregulado se hizo uso de la traducción al español de la escala breve del inventario motivated strategies for learning questionnaire (MSLQ) (Pintrich et al., 1991, 1993; Sharma, 2013), en la cual se presentan 24 preguntas correspondientes a las estrategias cognitivas y metacognitivas que utilizan los estudiantes en la regulación de sus actividades académicas. En este caso, las preguntas (p. ej., "Trato de relacionar mis ideas con lo que estoy aprendiendo en el curso de matemáticas") se responden en una escala Likert de siete puntos - de 1 (no es verdad para ti) a 7 (es muy verdadero para ti) - . Por último, esta escala reportó una consistencia interna aceptable para todo el instrumento $(\alpha=.73)$ y para sus respectivas subescalas: elaboración $(\alpha=.78)$, organización 
272

( $\alpha=.73)$, pensamiento crítico $(\alpha=.76)$ y autorregulación metacognitiva $(\alpha=.83)$ (Sharma, 2013).

Ahora bien, con excepción del inventario para evaluar la familia positiva, los demás instrumentos aplicados fueron traducidos del inglés al español por parte de un grupo de tres psicólogos investigadores bilingües del noroeste de México. Para un adecuado procedimiento de traducción de los instrumentos, primero se realizaron las traducciones de manera individual; posteriormente, se organizó un panel de revisión con un moderador con el fin de conseguir un total acuerdo en la traducción de cada uno de los ítems - donde se buscó usar las palabras más comunes al español para el contexto mexicano-; y, finalmente, se aceptaron las traducciones realizadas al alcanzar el $100 \%$ de acuerdo entre los traductores. Fue importante considerar como elementos esenciales en la traducción la experiencia en investigación de los traductores en temas relacionados con las escalas traducidas, así como el conocimiento de la cultura donde se realizaría la aplicación (Van de Vijver \& Hambleton, 1996).

Por último, todos los instrumentos fueron validados en un estudio piloto previo (no publicado), donde se comprobó la validez de constructo para el contexto mexicano mediante la aplicación de análisis factoriales confirmatorios. En la Tabla 1 se muestran los valores de alfa de Cronbach de las escalas utilizadas, donde cabe señalar que los valores obtenidos van de .84 — en la escala de amistades pro-académicas-a .92 - en la escala de aprendizaje autorregulado-, con lo cual se comprobó la pertinencia estadística y la aceptable consistencia interna de cada uno de los instrumentos aquí utilizados (Oviedo \& Arias, 2005).

\section{Procedimiento}

Inicialmente, una vez seleccionada la escuela donde se aplicarían los instrumentos, se explicó al personal directivo de la institución seleccionada los objetivos de la investigación, $\mathrm{y}$, tras la obtención de los permisos correspondientes, se preguntó sobre la cantidad de grupos de nuevo ingreso de la institución y sobre el número de estudiantes por grupo en promedio para decidir el número de grupos que se requería evaluar para completar el número de participantes necesario para la muestra.

Posteriormente, se realizó la selección de los grupos requeridos de manera aleatoria, y se acudió a los grupos seleccionados para explicar a los estudiantes y a los profesores los objetivos de la investigación, la confidencialidad de sus datos personales, y la necesidad de que firmaran su aceptación para participar libre y voluntariamente en el estudio. Por ser estudiantes menores de edad, se entregó a cada uno de ellos un consentimiento informado para que fuera firmado por sus padres o tutores. Estos consentimientos, tanto el personal como el de sus padres o tutores, se recogieron al día siguiente, y solamente participaron en la investigación los estudiantes que presentaron los dos consentimientos firmados.

Finalmente, la aplicación de los instrumentos se realizó grupalmente y en el horario de clases por dos psicólogos capacitados encargados. En este momento se cuidó que los participantes respondieran siguiendo las instrucciones que se indicaban en cada una de las escalas, sin brindarles ayudas adicionales. El tiempo aproximado para contestar los instrumentos fue de 50 minutos.

Tabla 1.

Valores alfa de Cronbach de las escalas usadas en la investigación

\begin{tabular}{lccccc}
\hline \multicolumn{1}{c}{ Escala } & Mín. & Máx. & Media & $D E$ & Alfa \\
\hline Amistades pro-académicas & 0 & 4 & 2.3 & 0.89 & .84 \\
Apoyo académico padres & 0 & 5 & 2.5 & 0.72 & .87 \\
Ambiente familiar positivo & 0 & 4 & 2.8 & 0.83 & .90 \\
Compromiso académico & 2 & 4 & 3.4 & 0.51 & .89 \\
$\quad$ Emocional & 1 & 5 & 3.7 & 0.57 & .86 \\
Conductual & 1 & 4 & 2.0 & 0.59 & .72 \\
Cognitivo & 2 & 5 & 4.4 & 1.02 & .72 \\
Aprendizaje autorregulado & 1 & 7 & 4.6 & 1.11 & .92 \\
$\quad$ Organización & 1 & 7 & 4.4 & 1.4 & .75 \\
Pensamiento crítico & 1 & 7 & 4.4 & 1.2 & .76 \\
Estrategias elaboración & 1 & 7 & 4.5 & 1.2 & .77 \\
Autorregulación metacognitiva & 2 & 7 & 4.9 & 1.1 & .77 \\
\hline
\end{tabular}




\section{Análisis de datos}

En un primer momento, se realizaron análisis descriptivos de los datos con el programa SPSS, v. 23; después, se efectuaron las pruebas de normalidad de los datos; se conformaron variables latentes con las sumatorias de los puntajes medios de cada uno de los ítems de las escalas; se realizó una correlación de Pearson entre las variables del estudio para medir el grado de asociación entre las variables del modelo estructural; y, finalmente, se probó el modelo de ecuaciones estructurales por medio del programa EQS, con relaciones directas e indirectas entre las variables de la investigación - donde se midió el grado de ajuste del modelo teórico e hipotético propuesto con los datos- (Bentler, 2006).

Posteriormente, para evaluar la validez compuesta del modelo estructural se comparó el modelo hipotético propuesto con el conjunto de los datos obtenidos en la investigación - por medio de los índices de ajuste del modelo- (Escobedo et al., 2016); y, teniendo en cuenta que los modelos de ecuaciones estructurales requieren de puntajes de bondades de ajuste adecuados, se esperó que el índice de a ajuste normado Bentler-Bonnet (BBNFI) y el índice de ajuste no normado Bentler-Bonnet fueran superiores a .90; así como que el índice de ajuste comparativo (CFI) obtuviera valores superiores a .95 ; además que hubiera relación entre $\chi^{2} / \mathrm{gl}$ menor o igual a 2.5 , así como un valor RMSEA menor de 0.06-0.08 (Bentler, 2006; Schreiber, 2008).

Por último, se conformó un factor de ambiente de aprendizaje positivo con las variables amistades pro-académicas, apoyo académico de padres y ambiente familiar positivo; el compromiso académico se evaluó como un solo factor con base en la propuesta teórica- que contempla el compromiso emocional, conductual y cognitivo (Appleton et al., 2006); y el aprendizaje autorregulado se probó con sus cuatro componentes: estrategias de elaboración, organización, pensamiento crítico y autorregulación metacognitiva (Sharma, 2013).

\section{Resultados}

Inicialmente, para evaluar la normalidad multivariada de los datos se utilizó la prueba del coeficiente de Mardia (1970), donde se encontró con un valor de 20.5; teniendo en cuenta que cuando dicho coeficiente es menor a $p(p+2)$ - donde $p$ son las variables observadas, que en el caso de la presente investigación fueron 10 -, es posible afirmar la normalidad de los datos (Bollen, 1989). Con este puntaje, se justifica la utilización de pruebas estadísticas para muestras normales, como el análisis de correlación de Pearson.

Ahora bien, como se muestra en la Tabla 2 -donde se presentan las correlaciones de Pearson de las variables de la investigación-, los resultados mostraron que todas las correlaciones fueron positivas y significativas; que la mayoría de las correlaciones tuvieron una $p<.01$; y que solamente se encontraron cinco correlaciones con una $p<.05-$ que fueron bajas-, entre ellas las correlaciones entre las variables de apoyo académico de amigos y compromiso académico conductual $(r=.19)$, entre apoyo académico de amigos y autorregulación académica de organización $(r=.16)$, entre apoyo académico de amigos y autorregulación académica de pensamiento crítico $(r=.18)$, y entre apoyo académico de amigos y autorregulación metacognitiva $(r=.17)$. En este punto resulta necesario ser cautos con la interpretación de las correlaciones significativas bajas $(<.36)$ en muestras grandes $(n>100)$, puesto que, por lo general, el coeficiente de Pearson tiende a ser significativo ante dichos niveles de asociación (Taylor, 1990). Para la interpretación más precisa de la relación entre las variables sería necesario aplicar análisis adicionales, como, por ejemplo, el análisis de ecuaciones estructurales, pues este utiliza la matriz de covarianza entre las variables para estimar el nivel de relación y el nivel de ajuste de los datos con respecto a las hipótesis (Bentler, 2006).

Por otra parte, en la Figura 2 se presenta el resultado del modelo estructural de la investigación, en el cual todos los pesos factoriales y coeficientes estructurales fueron significativos $(p<.05)$. Como se puede observar, el factor ambiente de aprendizaje positivo — conformado por las amistades pro-académicas, el apoyo académico de padres y el ambiente familiar positivo - se relacionó de manera alta y positivamente con el compromiso académico (.80); $\mathrm{y}$, a su vez, el compromiso académico mostró una correlación alta y positiva (.55) con el aprendizaje autorregulado. Asimismo, el compromiso académico se conformó por el compromiso conductual, emocional y cognitivo; mientras que el aprendizaje autorregulado se formó con las dimensiones propuestas por la teoría —estrategias de elaboración, organización, pensamiento crítico y autorregulación metacognitiva-

En general, el modelo presentó valores de bondad de ajuste superiores a .93 , valores de chi cuadrado no significativos, y valor RMSEA menor a .06, por lo cual se establece como un modelo estadísticamente aceptable y con apoyo empírico de los datos (Bentler, 2006). 
Tabla 2.

Correlaciones de Pearson entre las variables de la investigación

\begin{tabular}{|c|c|c|c|c|c|c|c|c|c|c|c|}
\hline & COMPEM & CAPEM & COMCB & COMCG & AUTRG & AUTOR & AUTPC & AUTAM & AUTE & AMFP & APAP \\
\hline COMPEM & 1 & & & & & & & & & & \\
\hline CAPEM & $.96 * *$ & 1 & & & & & & & & & \\
\hline COMCB & $.38 * *$ & $.38 * *$ & 1 & & & & & & & & \\
\hline COMCG & $.44 * *$ & $.47 * *$ & $.41 * *$ & 1 & & & & & & & \\
\hline AUTRG & $.32 * *$ & $.32 * *$ & $.33 * *$ & $.39 * *$ & 1 & & & & & & \\
\hline AUTOR & $.25^{* *}$ & $.25^{* *}$ & $.41 * *$ & $.34 * *$ & $.80 * *$ & 1 & & & & & \\
\hline AUTPC & $.33 * *$ & $.35 * *$ & $.32 * *$ & $.39 * *$ & $.89 * *$ & $.66^{* *}$ & 1 & & & & \\
\hline AUTAM & $.27 * *$ & $.26 * *$ & $.21 * *$ & $.31 * *$ & $.92 * *$ & $.62 * *$ & $.76^{* *}$ & 1 & & & \\
\hline AUTE & $.28 * *$ & $.30 * *$ & $.30 * *$ & $.36^{* *}$ & $.91 * *$ & $.68 * *$ & $.78 * *$ & $.78 * *$ & 1 & & \\
\hline AMFP & $.51 * *$ & $.53 * *$ & $.45^{* *}$ & $.37 * *$ & $.31 * *$ & $.35 * *$ & $.29 * *$ & $.26 * *$ & $.25 * *$ & 1 & \\
\hline APAP & $.50 * *$ & $.50 * *$ & $.35^{* *}$ & $.34 * *$ & $.38 * *$ & $.34 * *$ & $.31 * *$ & $.34 * *$ & $.35 * *$ & $.68 * *$ & 1 \\
\hline APDA & $.39 * *$ & $.42 * *$ & $.19 *$ & $.30 * *$ & $.21 * *$ & $.16^{*}$ & $.18^{*}$ & $.17 *$ & $.23 * *$ & $.38 * *$ & $.48 * *$ \\
\hline
\end{tabular}

Nota. COMPEM = compromiso académico emocional; CAPEM = compromiso académico emocional, cognitivo y conductual; $\mathrm{COMC}=$ compromiso académico conductual $\mathrm{COMCG}=$ compromiso académico cognitivo; $\mathrm{AUTRG}=$ autorregulación académica; AUTOR $=$ autorregulación académica de organización; AUTPC $=$ autorregulación académica de pensamiento crítico; AUTAM = autorregulación metacognitiva; AUTE = autorregulación académica de estrategias de elaboración; AMFP = ambiente familiar positivo; $\mathrm{APAP}=$ apoyo académico de padres; APDA = apoyo académico de amigos. ${ }^{* *} \mathrm{p}<.01 ;{ }^{*} \mathrm{p}<.05$.

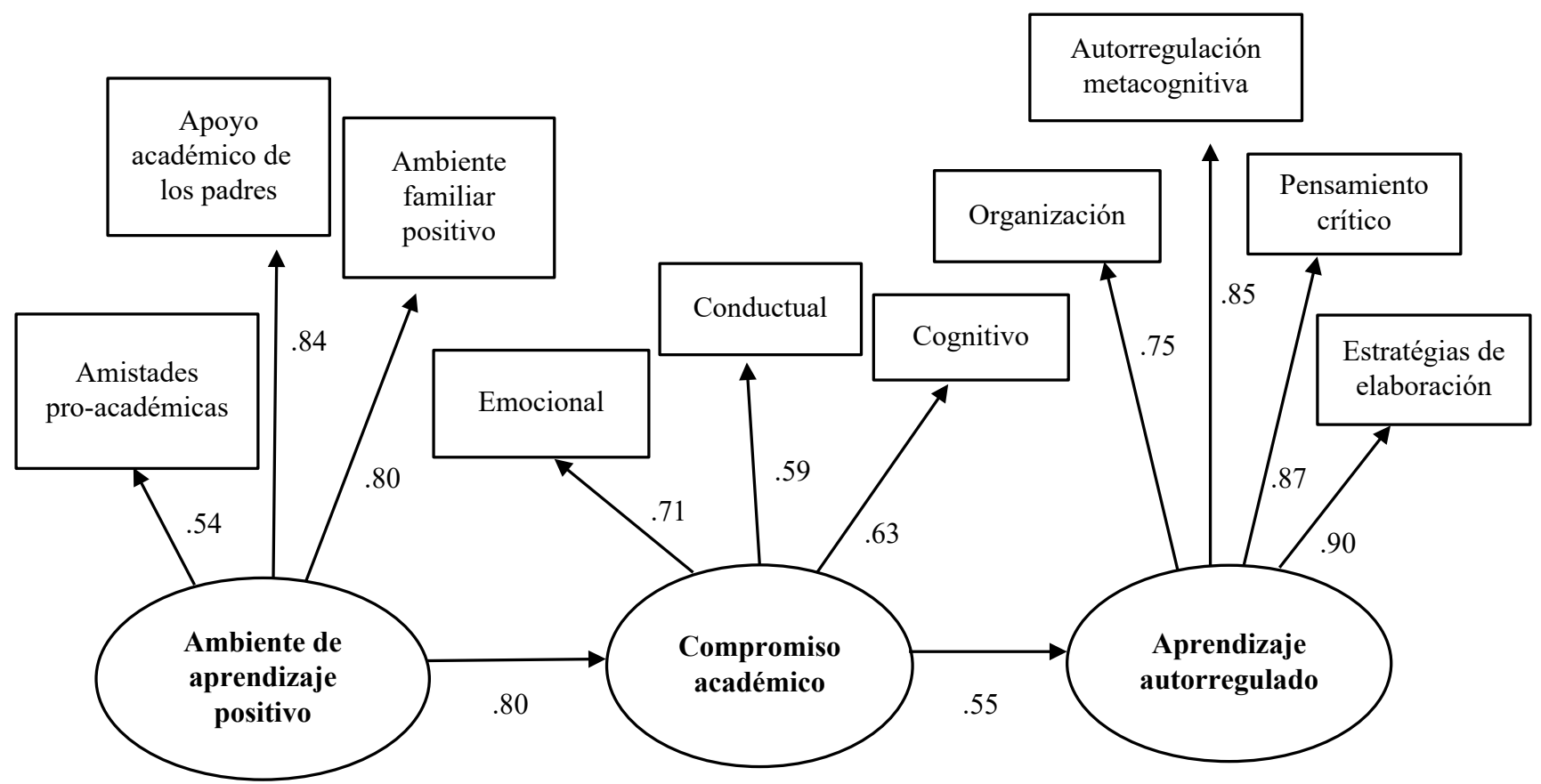

Figura 2. Modelo de aprendizaje autorregulado y ambiente de aprendizaje positivo de estudiantes de bachillerato. Todos los pesos factoriales y los coeficientes estructurales son significativos $(p<.05)$. Bondades de ajuste: $\chi^{2}=53.9(30 \mathrm{gl})$, $p=0.00463, \mathrm{BBNFI}=0.93, \mathrm{BBNNFI}=0.95, \mathrm{CFI}=0.97, \mathrm{RMSEA}=0.07, R^{2}$ (aprendizaje autorregulado $)=0.30$. 


\section{Discusión}

El propósito de la presente investigación fue evaluar la relación entre el ambiente de aprendizaje positivo, el compromiso académico y el aprendizaje autorregulado en estudiantes de bachillerato de Hermosillo, México. De acuerdo con los resultados, el compromiso académico, como se esperaba en la hipótesis inicial, resultó ser una variable mediadora de la relación entre el ambiente de aprendizaje positivo y el aprendizaje autorregulado.

En particular, el ambiente de aprendizaje positivo se conformó por las amistades pro-académicas, el apoyo académico de los padres y el ambiente familiar positivo, de modo que mantenerse con un compromiso académico en el bachillerato, de acuerdo con el modelo de ecuaciones estructurales resultante, requiere del apoyo emocional y material otorgado por los padres y los amigos, tal como lo señalan otras investigaciones (Álvarez et al., 2015; Shin $\&$ Ryan, 2014). De hecho, de acuerdo con la literatura, el interés mostrado por los padres en el proceso educativo de sus hijos, manifestado por su involucramiento en las tareas y proyectos escolares, mejora las variables asociadas al rendimiento académico de los estudiantes adolescentes (Fajardo et al., 2017); sumado a que el apoyo académico recibido por las amistades pro-académicas facilita que los estudiantes se sientan socialmente conectados con un grupo, lo que puede incrementar sus emociones positivas hacia las actividades académicas (Rodríguez et al., 2018) y afectar positivamente su motivación y persistencia académica (Ricard \& Pelletier, 2016).

Adicional a esto, con base en los resultados, un ambiente familiar positivo, donde los miembros cooperan para el mantenimiento de la estabilidad y positividad del contexto, permite que los miembros convivan en un entorno organizado y cooperativo que puede beneficiar el desarrollo de comportamientos responsables en el ámbito escolar - como el compromiso académico emocional, conductual y cognitivo-, lo cual sirve para corroborar la teoría de ambientes positivos como promotores de adaptación psicológica en el ambiente educativo (Corral et al., 2014).

Asimismo, los resultados del modelo también mostraron que el compromiso académico correlacionó positivamente con el aprendizaje autorregulado, el cual, según los resultados de la presente investigación, incluye las dimensiones de organización, pensamiento crítico, estrategias de elaboración y autorregulación metacognitiva, tal como lo afirmó Sharma (2013) en su investigación. En este sentido, según Lam et al. (2012), los estudiantes más comprometidos son capaces de seleccionar metas de aprendizaje y, al cumplirlas, se aumenta su capacidad de autogestión del aprendizaje, lo que se relaciona, finalmente, con su aprendizaje autorregulado (Skinner \& Pitzer, 2012; You \& Kang, 2014).

Por otra parte, como mencionan Corral et al. (2014), los ambientes positivos representan escenarios de transacción donde las personas reciben y obtienen beneficios, pero dan algo a cambio, lo que posibilita la permanencia del sistema. De esta manera, un ambiente de aprendizaje positivo posibilita que los estudiantes "reciban" los beneficios de un entorno que proporciona los requerimientos esenciales - materiales y emocionales - para el efectivo aprendizaje, mientras que los estudiantes "regresan" la inversión que realizaron las personas que otorgaron los apoyos mediante su compromiso académico, el cual se relaciona, finalmente, con su aprendizaje autorregulado.

Ahora, al ser el factor de ambiente positivo de aprendizaje una variable exógena, esta misma constituye el aspecto central que probablemente requiere atenderse en programas cuyo objetivo sea incrementar el compromiso académico y el aprendizaje autorregulado de los estudiantes de bachillerato como medio para mantener su permanencia exitosa y prevenir el abandono escolar - lo cual requiere comprobarse en estudios prospectivos futuros- - . Teniendo esto en cuenta, los resultados del presente estudio pueden servir como base para que en las intervenciones psicoeducativas dirigidas a mejorar la autorregulación de los estudiantes de bachillerato se promueva que los estudiantes con mayor éxito académico se relacionen y apoyen académicamente a aquellos que muestren algún tipo de rezago educativo. Por otra parte, en dichas intervenciones también se requerirá incluir la promoción de las interacciones positivas al interior de la familia de los estudiantes, donde se resalte la importancia de mantener los apoyos académicos materiales y emocionales. Además, la dinámica de los apoyos académicos otorgados por las amistades y la familia pueden generar un mayor bienestar psicológico en quienes los brindan y los reciben, lo que puede ayudar al mantenimiento del entorno positivo sustentable.

A partir de lo anterior, las investigaciones futuras deberían evaluar el resultado del bienestar psicológico en los ambientes escolares positivos junto con la medición de alguna variable de ajuste escolar, como las calificaciones escolares o la culminación con éxito de los programas académicos de los estudiantes que reciben los apoyos. En este sentido, los estudios longitudinales subsiguientes podrán comprobar, finalmente, la hipótesis de que los ambientes positivos escolares pueden reducir la deserción escolar de los estudiantes en riesgo.

Por otro lado, el modelo que resultó de la presente investigación explicó casi una tercera parte de la varianza de la autorregulación académica de los estudiantes de 
bachillerato evaluados, por lo cual resulta importante seguir investigando el papel de los ambientes de aprendizaje positivo y el compromiso académico en su relación con el aprendizaje autorregulado; sin embargo, también podría profundizarse en el estudio de otras variables psicológicas que puedan participar en la explicación del resto de la varianza del aprendizaje autorregulado, como lo son las funciones ejecutivas del lóbulo frontal, las cuales abarcan como una capacidad ejecutiva fundamental la autorregulación del comportamiento (Franklin et al., 2018). Lo anterior requiere ser abordado por sucesivas investigaciones.

Finalmente, el presente estudio no carece de limitaciones, y entre ellas resaltan, por ejemplo, que no es posible realizar generalizaciones de los resultados debido a que el estudio fue de tipo correlacional transversal, y que no se evaluó la relación del compromiso académico o el aprendizaje autorregulado con alguna medida específica de rendimiento académico, como con los puntajes personales obtenidos en la prueba PLANEA, donde se podría esperar alguna relación positiva — esto podrá ser corroborado en investigaciones posteriores- - Sin embargo, a pesar de las limitaciones señaladas, el presente estudio presenta un acercamiento a los factores de tipo ambiental - denominados ambientes de aprendizaje positivos - que se relacionan con variables psicológicas evidenciadas por los estudiantes académicamente exitosos.

En conclusión, se podría afirmar, a manera de hipótesis, que es probable que los ambientes de aprendizajes positivos sean factores protectores del abandono escolar de estudiantes de bachillerato; aspecto que debería estudiarse en trabajos longitudinales a largo plazo. Asimismo, se sugiere que las investigaciones futuras sobre el tema incrementen la muestra a otros bachilleratos con diferente nivel de aprovechamiento, y que, a la vez, realicen un seguimiento de tipo longitudinal de las variables del estudio para obtener un modelo estructural explicativo generalizable a otras poblaciones de estudiantes de bachillerato. Incluso, se propone medir el grado de rendimiento académico alcanzado por los estudiantes con mayor aprendizaje autorregulado y compararlo con el rendimiento académico de los estudiantes con menores puntajes de dicha variable, para así, finalmente, evidenciar la relación entre las variables estudiadas y la eficiencia terminal de los estudiantes de bachillerato.

\section{Referencias}

Álvarez, A., Suárez, N., Tuero, E., Núñez, J. C., Valle, A., \& Regueiro, B. (2015). Implicación familiar, autoconcepto del adolescente y rendimiento académico. European
Journal of Investigation in Health, 5(3), 293-311. https:// doi.org/10.1989/Ejihpe.V5i3.133

Appleton, J. J., Christenson, S. L., Kim, D., \& Reschly, A. L. (2006). Measuring cognitive and psychological engagement: Validation of the student engagement instrument. Journal of School Psychology, 44(5), 427-445. https://doi. org/10.1016/j.jsp.2006.04.002

Aranda, C. C., Gaxiola, R. J., González, L. S., \& Valenzuela, H. E. (2015). Construcción y validación de una escala de ambiente familiar. Memorias del XXIII Congreso Mexicano de Psicología, 610-613.

Bentler, P. M. (2006). EQS 6 Structural Equations Program Manual. Mulivariate Software Inc.

Bollen, K. A. (1989). Structural equations with latent variables. John Wiley \& Sons.

Chase, P., Hilliard, L., Geldhof, J., Warren, D. J., \& Lerner, R. (2014). Academic achievement in the high school years: The changing role of school engagement. Journal of Youth Adolescence, 43(6), 884-896. https://doi.org/10.1007/ s10964-013-0085-4

Chen, J. J. (2005). Relation of academic support from parents, teachers, and peers to Hong Kong adolescents' academic achievement: The mediating role of academic engagement. Genetic, social, and general psychology monographs, 131(2), 77-127. https://doi.org/10.3200/ MONO.131.2.77-127

Chen, J. J. (2008). Grade-level differences relations of parental, teacher and peer support to academic engagement and achievement among Hong Kong students. School psychology international, 29(2), 183-198. https://doi. org/10.1177/0143034308090059

Corral, V. V., Frías, A. M., Gaxiola, R. J., Fraijo, S. B., Tapia, F. C., \& Corral, F. N. (2014). Ambientes positivos: ideando entornos sostenibles para el bienestar humano y la calidad ambiental. Pearson \& UNISON.

Daura, F. T. (2015). Aprendizaje autorregulado y rendimiento académico en estudiantes del ciclo clínico de la carrera de Medicina. Revista Electrónica de Investigación Educativa, 17(3), 28-45. http://www.redalyc.org/articulo. oa? id $=15540997003$

Eccles, J. S., \& Harold, R. D. (2013). Family involvement in children's and adolescents' schooling. En A. Booth \& J. F. Dunn (Eds.), Family-school links: How do they affect educational outcomes? (pp. 3-34). Routledge.

Escobedo, P. M., Hernández, G. J., Estebané, O. V., \& Martínez, M. G. (2016). Modelos de ecuaciones estructurales: características, fases, construcción, aplicación y resultados. Ciencia \& trabajo, 18(55), 16-22. https://scielo.conicyt.cl/ pdf/cyt/v18n55/art04.pdf

Fajardo, B. F., Maestre, C. M., Felipe, C. E., León del Barco, B., \& Polo del Río, M. I. (2017). Análisis del rendimiento académico de los alumnos de educación secundaria obligatoria según las variables familiares. Educación $X X 1$, 20(1), 209-232. http://e-spacio.uned.es/fez/eserv/bibliun 
ed:EducacionXXI-2017-20-1-5045/Analisis_rendimien to academico.pdf

Fooladvand, K., Soltani, M., Fathi Ashtiani, A., \& Shoae, Z. (2012). Psychometric properties of Tinio Students' engagement in the task. Educational Measurement Quarterly, 3(8), 155-182.

Franklin, P., Tsujimoto, K. C., Lewis, M. E., Tekok-Kilic, A., \& Frijters, J. C. (2018). Sex differences in self-regulatory executive functions are amplified by trait anxiety: The case of students at risk for academic failure. Personality and Individual Differences, 129, 131-137. https://doi. org/10.1016/j.paid.2018.03.019

Fredericks, J. A., Blumenfeld, P. C., \& Paris, A. H. (2004). School engagement: Potential of the concept, state of the evidence. Review of Educational Research, 74(1), 59-109. https://doi.org/10.3102/00346543074001059

Gaxiola, R. J., \& González, S. (2019). Apoyo percibido, resiliencia, metas y aprendizaje autorregulado en bachilleres. Revista Electrónica de Investigación Educativa, 21(e08), 1-10. https://doi.org/10.24320/redie.2019.21.e08.1983

Gaxiola, R. J., Gaxiola, V. E., Escobedo, H. P., \& González, L. S. (2018). Variables relacionadas con la resiliencia o adaptabilidad académica de bachilleres. En L. R. Díaz, L. L. Reyes \& R. F. López (Eds.), La psicología social en México (Vol. XVII, pp. 491-507). Editorial Asociación Mexicana de Psicología Social.

Gómez, P., Pérez, C., Parra, P., Ortiz, L., Matus, O., McColl, P., ... Meyer, A. (2015). Relación entre el bienestar y el rendimiento académico en alumnos de primer año de medicina. Revista Médica de Chile, 143(7), 930-937. https://doi. org/10.4067/S0034-98872015000700015

Instituto Nacional para la Evaluación de la Educación [INEE] (2017). La Educación obligatoria en México. Informe 2017 (1. ${ }^{a}$ Ed.). INEE. http://publicaciones.inee.edu.mx/busca dorPub/P1///242/P1I242.pdf

King, R. B., \& Ganotice, F. A. (2014). The social underpinnings of motivation and achievement: Investigating the role of parents, teachers, and peers on academic outcomes. The Asia-Pacific Education Researcher, 23(3), 745-756. https:// doi.org/10.1007/s40299-013-0148-Z

Lam, S. F., Wong, B. P., Yang, H., \& Liu, Y. (2012). Understanding student engagement with a contextual model. En S. L., Christenson, A. L., Reschly, \& C. Wylie (Eds.), Handbook of research on student engagement (pp. 403-419). Springer.

Lamas, H. (2008). Aprendizaje autorregulado, motivación y rendimiento académico. Liberabit, 14(14), 16-20. http://www.scielo.org.pe/scielo. php?pid=S1729-48272008000100003\&script=sci_arttext

Mardia, K. V. (1970). Measures of multivariate skewness and kurtosis with applications. Biometrika, 57(3), 519-530.

Oviedo, H. C., \& Arias, A. C. (2005). Aproximación al uso del coeficiente alfa de Cronbach. Revista Colombiana de Psiquiatría, 34(4), 572-580. http://www.redalyc.org/ pdf/806/80634409.pdf

Pintrich, P. R., Smith, D., Garcia, T., \& McKeachie, W. (1991). A manual for the use of the Motivated Strategies for Learning Questionnaire (MSLQ). The University of Michigan.

Pintrich, P. R., Smith, D., Garcia, T., \& McKeachie, W. (1993). Reliability and Predictive validity of the Motivated Strategies for Learning Questionnaire (MSLQ). Education Psychology Measurement, 53(3), 801-813. https://doi. org $/ 10.1177 / 0013164493053003024$

Ricard, N. C., \& Pelletier, L. G. (2016). Dropping out of high school: The role of parent and teacher self-determination support, reciprocal friendships and academic motivation. Contemporary Educational Psychology, 44, 32-40. https:// doi.org/10.1016/j.cedpsych.2015.12.003

Rodríguez, F. A., Ramos, D. E., Ros, I., \& Zuazagoitia, A. (2018). Implicación escolar de estudiantes de secundaria: $\mathrm{La}$ influencia de la resiliencia, el autoconcepto y el apoyo social percibido. Educación XX1, 21(1), 87-108, https://doi. org/10.5944/educXX1.16026

Roux, R. (2015). Estrategias de aprendizaje y su relación con el rendimiento académico en estudiantes de una escuela privada de educación media superior. Revista Actualidades Investigativas en Educación, 15(1), 1-16. https://doi. org/10.15517/aie.v15i1.17731

Schreiber, J. B. (2008). Core reporting practices in structural equation modeling. Research in social and administrative pharmacy, 4(2), 83-97. https://doi.org/10.1016/j. sapharm.2007.04.003

Seligman, M. E., \& Csikszentmihalyi, M. (2000). Positive psychology: An introduction. American psychologist, 55(1), 5-14. http://www.bdp-gus.de/gus/Positive-PsychologieAufruf-2000.pdf

SecretaríadeEducaciónPública[SEP](2017).PlanNacionalpara la Evaluación de los Aprendizajes (PLANEA) de Educación Media Superior. Instituto Nacional para la Evaluación de la Educación. https://www.gob.mx/sep/prensa/ comunicado-481-publica-sep-resultados-de-planea-2016

Sharma, A. (2013). Associations between self-efficacy beliefs, self-regulated learning strategies, and students' performance on model eliciting tasks: An examination of direct and indirect effects (tesis doctoral). University of Florida, California. https://eric.ed.gov/?id=ED558272

Shin, H., \& Ryan, A. (2014). Friendship networks and achievement goals: An examination of selection and influence processes and variations by gender. Journal of Youth Adolescence, 43(9), 1453-64. https://doi.org/10.1007/ s10964-0132-9

Skinner, E. A., \& Pitzer, J. R. (2012). Developmental dynamics of student engagement, coping, and everyday resilience. En S. L. Christenson, A. L. Reschly, \& C. Wyle (Eds.), Handbook of Research on Student Engagement (pp. 21-44). Springer. 
Soper, D. S. (2018). A-priori Sample Size Calculator for Structural Equation Models [Software]. https://www.da nielsoper.com/statcalc/calculator.aspx $? \mathrm{id}=89$

Taylor, R. (1990). Interpretation of the correlation coefficient: a basic review. Journal of diagnostic medical sonography, 6(1), 35-39. https://journals.sagepub.com/doi/ pdf/10.1177/875647939000600106

Van de Vijver, F., \& Hambleton, R. K. (1996). Traslating test: Some practical guidelines. European Psychologist, 1(2), 89-99. https://doi.org/10.1027/1016-9040.1.2.89

Wang, M. T., \& Eccles, J. S. (2012). Social support matters: Longitudinal effects of social support on three dimensions of school engagement from middle to high school. Child Development, 83(3), 877-895. https://doi. $\operatorname{org} / 10.1111 / \mathrm{j} .1467-8624.2012 .01745 . x$
Yazzie-Mintz, E. (2009). Report: Engaging the voice of students: A report on the 2007 y 2008 high school survey of student engagement. Center for Evaluation and Education Policy (CEEP) at Indiana University. http://www.niusi leadscape.org/lc/Record/223

You, J. W., \& Kang, M. (2014). The role of academic emotions in the relationship between perceived academic control and self-regulated learning in online learning. Computers \& Education, 77, 125-133. https://doi.org/10.1016/j. compedu.2014.04.018

Zimmerman, B. J. (2000). Attaining self-regulation: A social cognitive perspective. En M. Boekaerts, P. Pintrich \& M. Ziedner (Eds.), Handbook of self-regulation (pp. 13-39). Academic Press.

Zimmerman, B. J. (2002). Becoming a self-regulated learner: An overview. Theory into practice, 41(2), 64-70. http:// www.johnnietfeld.com/uploads/2/2/6/0/22606800/zimmer man_2002.pdf 